\title{
Testicular Metastasis from Urothelial Carcinoma of the Renal Pelvis: A Rare Case and Review of the Literature
}

\author{
Xue-wu Wu (D) ${ }^{1, *}$ \\ Yang Zhang ${ }^{2, *}$ \\ Yu-feng Li ${ }^{1}$ \\ Ye $\mathrm{Li}^{1}$ \\ Xiang-xiang Zhang' \\ Wei Wang ${ }^{\prime}$ \\ Jun-hai Ma' \\ Pei-long Wang' \\ Zhi-chun Dong' \\ Wei Shi' \\ Jun-qiang Tian'
}

'Department of Urology, The Second Hospital of Lanzhou University, Key

Laboratory of Urological Diseases in Gansu

Province, Gansu Nephro-Urological

Clinical Center, Lanzhou 730000, People's

Republic of China; ${ }^{2}$ Department of

Ultrasound in Medicine, Sixth People's

Hospital Affiliated to Shanghai Jiao Tong

University, Shanghai Institute of Ultrasound

in Medicine, Shanghai 200233, People's

Republic of China

*These authors contributed equally to this work

Correspondence: Jun-qiang Tian Department of Urology, The Second

Hospital of Lanzhou University,

Cuiyingmen 82, Chengguan District,

Lanzhou 730030, People's Republic of

China

Email tjq007263@sina.com
This article was published in the following Dove Press journal:

OncoTargets and Therapy

\begin{abstract}
This paper presents an extremely rare case of testicular metastasis arising from renal pelvis carcinoma. The testicle is a rare site of clinically detectable tumor metastasis, originating rarely from upper tract urothelial carcinoma (UTUC). There are only two cases concerning UTUC metastasis to the testis available in the literature. In this report, we presented a patient who developed serial testicle, lung, liver and retroperitoneal lymph node metastasis from primary urothelial carcinoma of the renal pelvis within one year after surgery and chemotherapy. In conclusion, for patients with a history of UTUC who present with testicular symptoms, clinicians should be highly alert for the possibility of malignant involvement at this site.
\end{abstract}

Keywords: urothelial carcinoma, renal pelvis, testicular metastasis

\section{Introduction}

Renal pelvis carcinoma occurs in the pelvis and calyx, and the incidence ranks second among renal tumors following kidney cancer. Transitional cell carcinoma is the most common histologically malignant urothelial carcinoma subtype of the renal pelvis. Two-thirds of upper tract urothelial carcinomas are at an invasive stage when diagnosed. There is a high multiple-organ metastatic recurrence rate at different sites, such as the lung, liver, bone and pelvic lymph nodes. ${ }^{1,2}$ Metastasis of UTUC to the testicles is extremely rare. Herein, we report a case of a renal pelvis carcinoma with serial testicular, lung, liver and retro-peritoneal lymph node metastasis diagnosed during follow-up following radical nephroureterectomy (with bladder cuff).

\section{Case Report}

In November 2015, a 57-year-old Chinese man was referred to our hospital with a history of progressive pain and discomfort of the left testicle, accompanied by intermittent lower back pain and loss of appetite over the previous 3 months. Physical examination showed a mass in the left testicle, together with swelling and tenderness. The skin of his scrotum was normal. Ultrasonography revealed a solid, hypoechoic, ill-defined and irregular mass in the left testicle, measuring approximately $35 \times 17 \mathrm{~mm}$ (Figure 1A), larger than in the exam taken in August 2015, where it was approximately $13 \times 9 \mathrm{~mm}$ (Figure 1B). Serum biomarkers, including $\alpha$-fetoprotein (AFP), $\beta$-human chorionic gonadotropin ( $\beta$-HCG), and lactate dehydrogenase (LDH), 

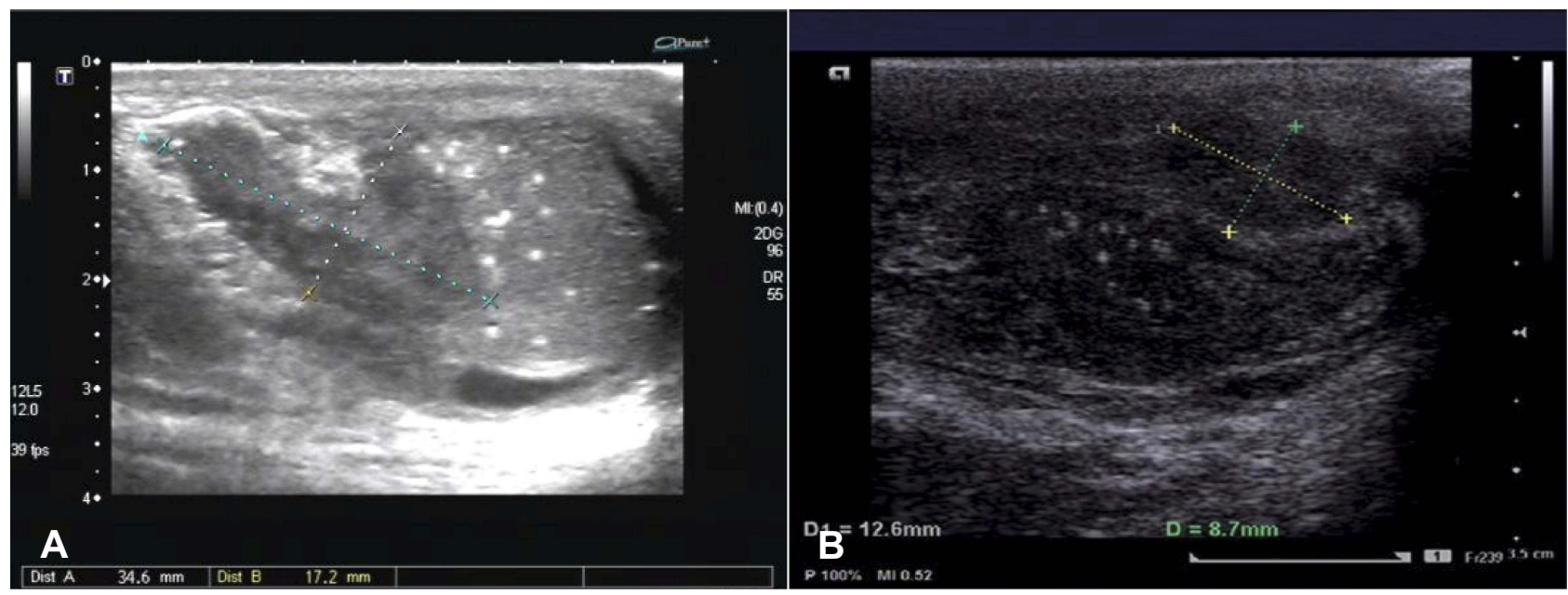

Figure I Tumor revealed by ultrasound. (A) A scrotal ultrasonic examination suggested a solid, hypoechoic, ill-defined and irregular mass in the left testicle, measuring $35 \times$ $17 \mathrm{~mm}$, November 20I5; (B) The testicular lesion had been diagnosed by scrotal ultrasonography in August 2015, which revealed a solid lesion in the left testis, measuring $13 \times 9 \mathrm{~mm}$.

were all normal, but with slightly elevated carbohydrate antigen 19-9 (CA19-9) and carcinoembryonic antigen (CEA). Inguinal exploration and radical orchiectomy were performed. Postoperative histopathological examination (HE) revealed irregularly cellular morphology, elevated karyokinesis and nuclear heterogeneity (Figure 2A). By immunohistochemistry (IHC), the tumor cells were diffusely reactive for pancytokeratin (CK) 5/6, CK7 (Figure 2B),
GATA3 (Figure 2C) and p40 (Figure 2D), but were not immunoreactive for Vimentin, AFP, placental-like alkaline phosphatase (PLAP), CD30, CD117, CD20, D2-40, octamer3/4 (Oct-3/4) and spalt-like transcription factor 4 (SALL4). The expression of Ki67 showed strong nuclear staining in $45 \%$ of the tumor cells. CK 7, GATA3 and p 40 , as biomarkers in transitional cell carcinoma, showed consistent expression in urothelial carcinoma. No evidence of carcinoma in situ

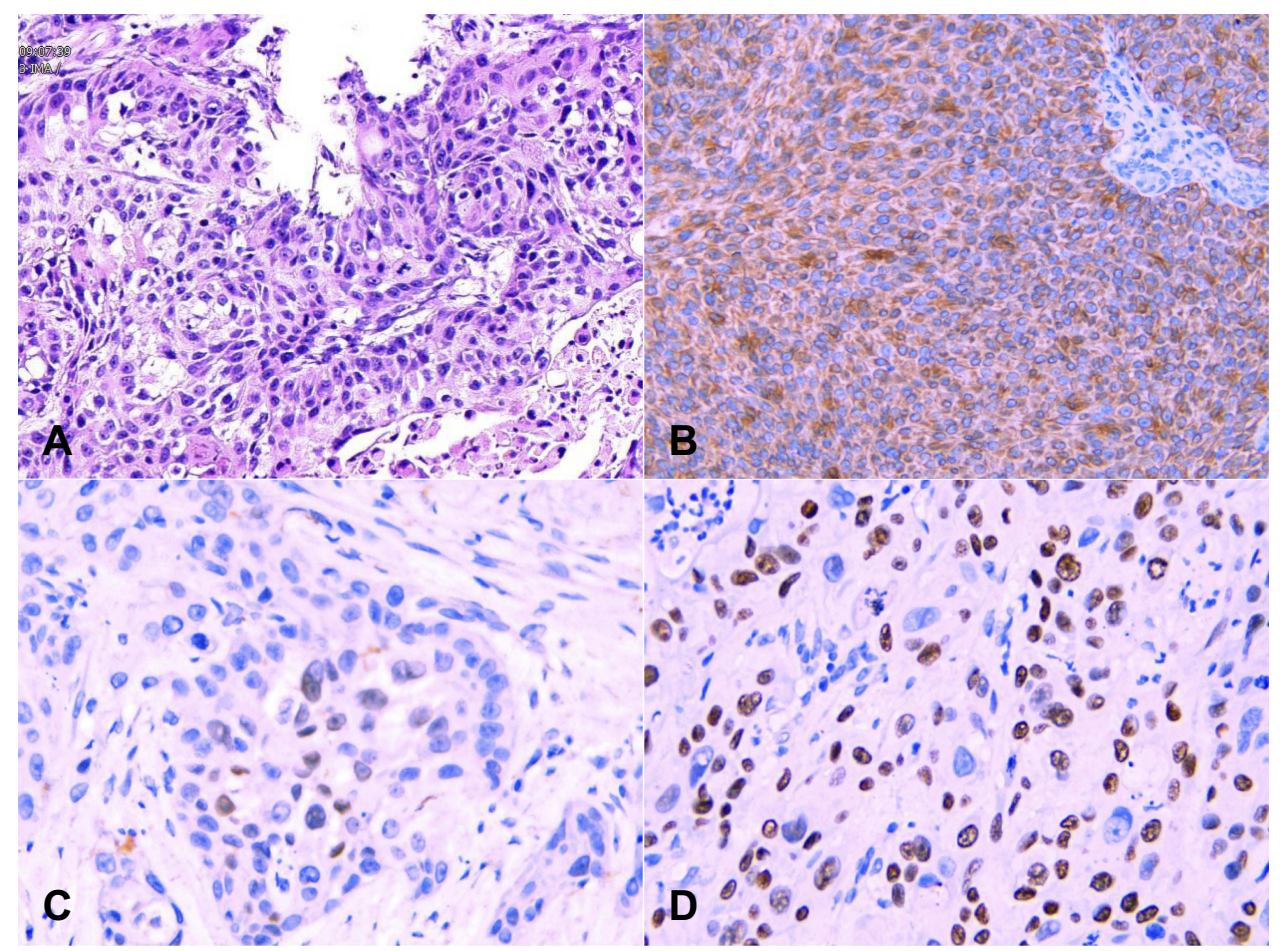

Figure 2 Pathological results in November 2015. Pathological results of testicular carcinoma. (A) Hematoxylin-eosin staining revealed irregular morphology, elevated karyokinesis and nuclear heterogeneity; (B-D) IHC demonstrated that the tumor cells were diffusely reactive for CK7, GATA3 and P40. 
was observed. Based on medical history and pathological examination, the patient was diagnosed with testicular metastasis from urothelial carcinoma of the renal pelvis. Following radical orchiectomy, the patient refused further treatment considering the intolerance to chemotherapy and passed away in December 2015.

By reviewing his medical history and radiological imaging, the patient was diagnosed with urothelial carcinoma of the left renal pelvis (T3N1M0) in April 2015 (Figure 3A-C). Radical nephroureterectomy was performed in our department. The tumor removed was approximately $60 \times 50$ $\times 50 \mathrm{~mm}$ in size, located in the pelvis wall of the left kidney, and a high level of invasive urothelial carcinoma of the renal pelvis was reported by pathologic examination, accompanied by necrosis and infiltration to the renal parenchyma, renal capsule and peripheral adipose tissues (Figure 3D). Postoperative HE revealed irregular cellular morphology, elevated karyokinesis and nuclear heterogeneity (Figure 4A). The tumor cells were diffusely positive for CK8/18, CK7 (Figure 4B), GATA3 (Figure 4C) and p40 (Figure 4D), EMA and p53 but negative for Vimentin, CD10, and CK20, and the expression of Ki67 showed strong nuclear staining over $45 \%$ of the neoplastic cells. No other specific past medical history was presented. Gemcitabine plus nedaplatin were administered in the oncology department of our hospital for 4 cycles after the operation.

Subsequently, in August 2015, the patient suffered from left testicular pain and discomfort without obvious inducement. Physical examination showed a nodulus in the left testicle accompanied by tenderness, and the scrotal skin was normal. Ultrasonography showed a $13 \times 9 \mathrm{~mm}$ hypoechoic lesion (Figure 1B) in the superior albuginea of the left testicle. Enhanced computed tomography (CT) showed that there were metastatic nodules in the right inferior lobe (Figure 5A), diffuse enlargement of retroperitoneal lymph nodes (Figure 5B), space-occupying lesions in the liver (Figure 5C) and metastatic lymph nodes in the mediastinum. The bone scan was unremarkable. These images indicated metastasis of secondary testicle, lung, liver, retroperitoneal and mediastinal lymph nodes. No corresponding symptoms, such as chest pain and hoarseness, were shown in these systems. The patient refused further treatment due to a tolerance to the testicular symptoms and an intolerance to chemotherapy.

\section{Consent}

This study was approved by the Institutional Review Board of Lanzhou University Second Hospital. The patient's daughter provided written informed consent and gave permission for the use of biopsies and the publication of case details, including images.

\section{Discussion}

Herein, we presented a patient who developed metastasis to the testicle, lung, liver and retroperitoneal lymph node from urothelial carcinoma of the renal pelvis. Lung metastasis is quite common, while secondary neoplasia of testicular cancers from solid tumors, except for leukemia and lymphoid carcinoma, is extremely rare, accounting for only $0.02 \%$ to $3.6 \%$ of all testicular neoplastic lesions. ${ }^{3}$ Metastatic involvement of the testicle by UTUC is even rarer. Testicular metastasis is generally found occasionally during orchiectomy for prostate cancer or at autopsy. ${ }^{4}$ Other than leukemia and lymphoma, the most common origins of secondary testicular tumors
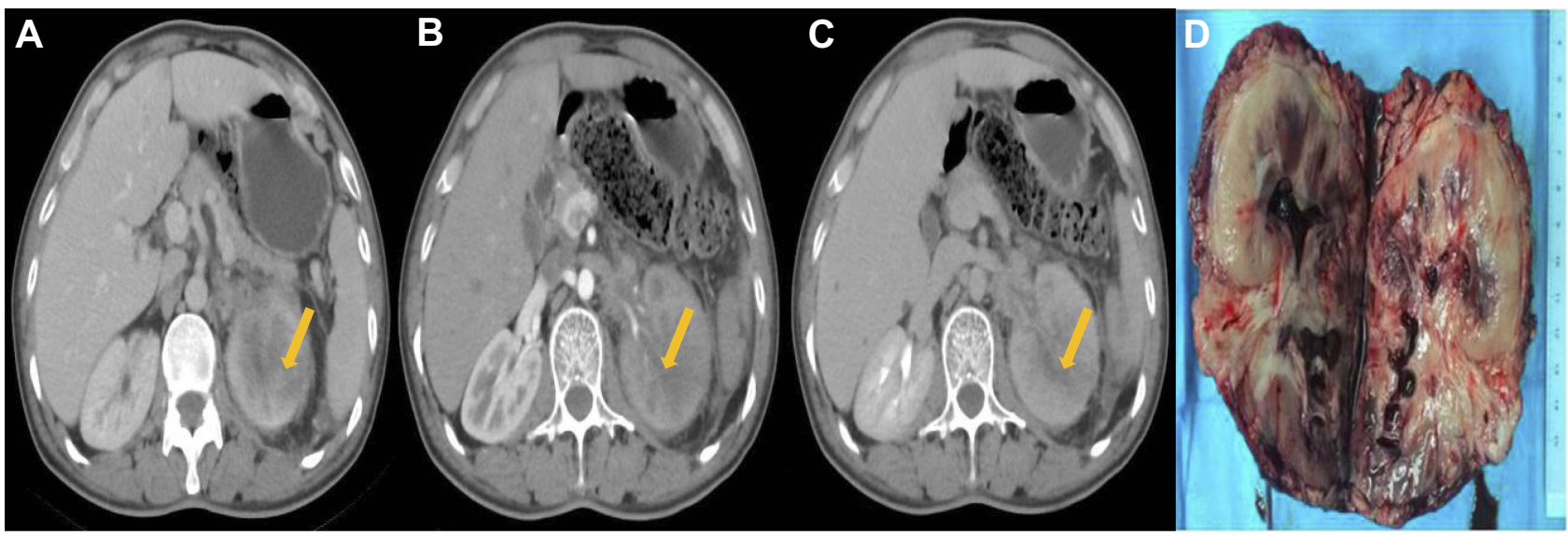

Figure 3 Primary lesion revealed in April 2015. The first three images are preoperative CT findings of left renal pelvis lesions. (A) Plain scan CT, (B) Enhanced CT arterial phase, (C) Enhanced CT Excretion Period and (D) Tumor resected, sized $60 \times 50 \times 50 \mathrm{~mm}$, located in the left renal pelvic wall, accompanied by necrosis, invading the renal parenchyma, renal capsule and perirenal adipose tissue. 


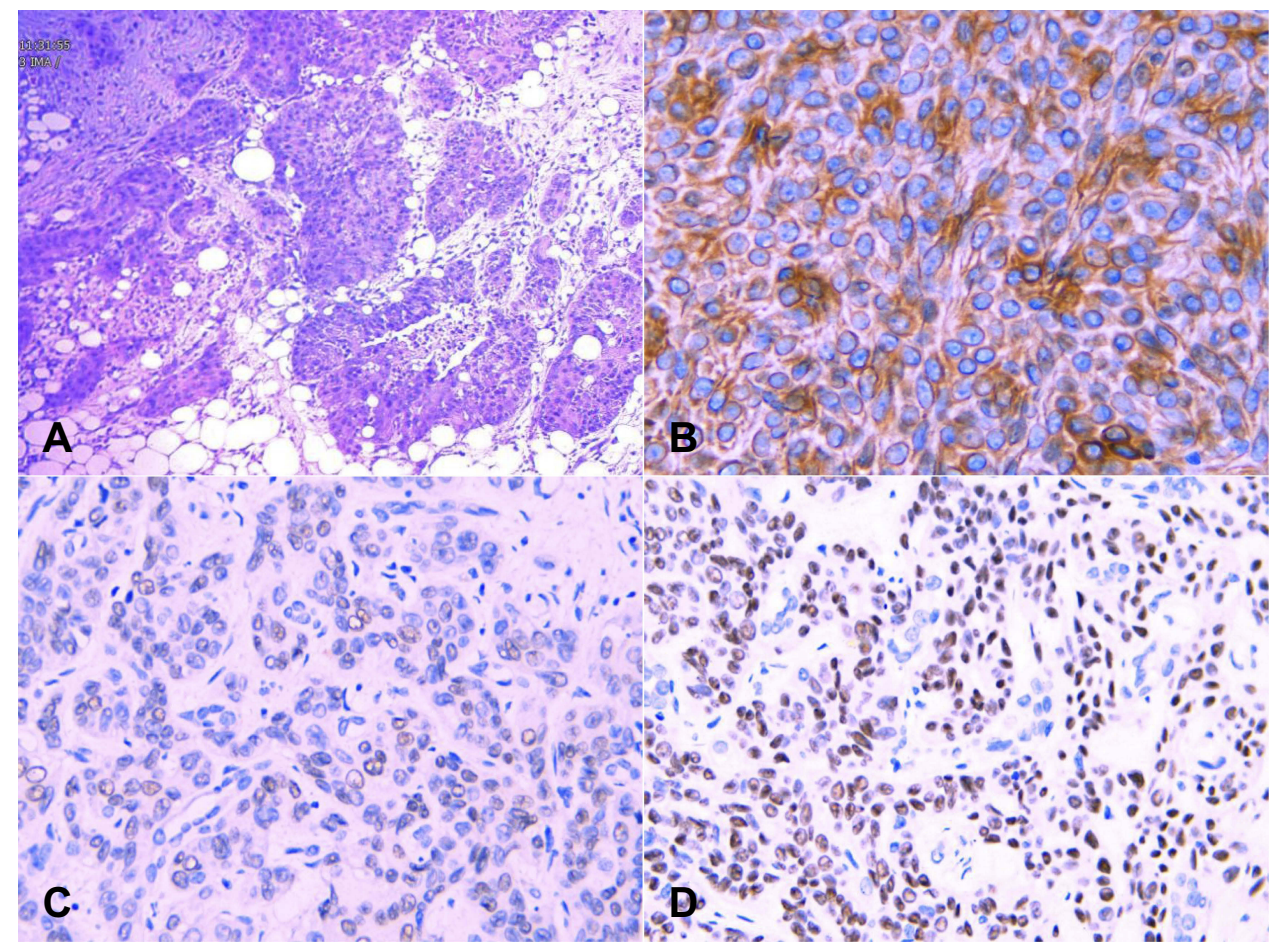

Figure 4 Pathological results in April 2015. Pathological results of renal pelvis carcinoma. (A) HE staining revealed an irregular morphology, elevated karyokinesis and nuclear heterogeneity; (B-D) IHC revealed that CK7, GATA3 and p40 were diffusely reactive in the tumor cells.

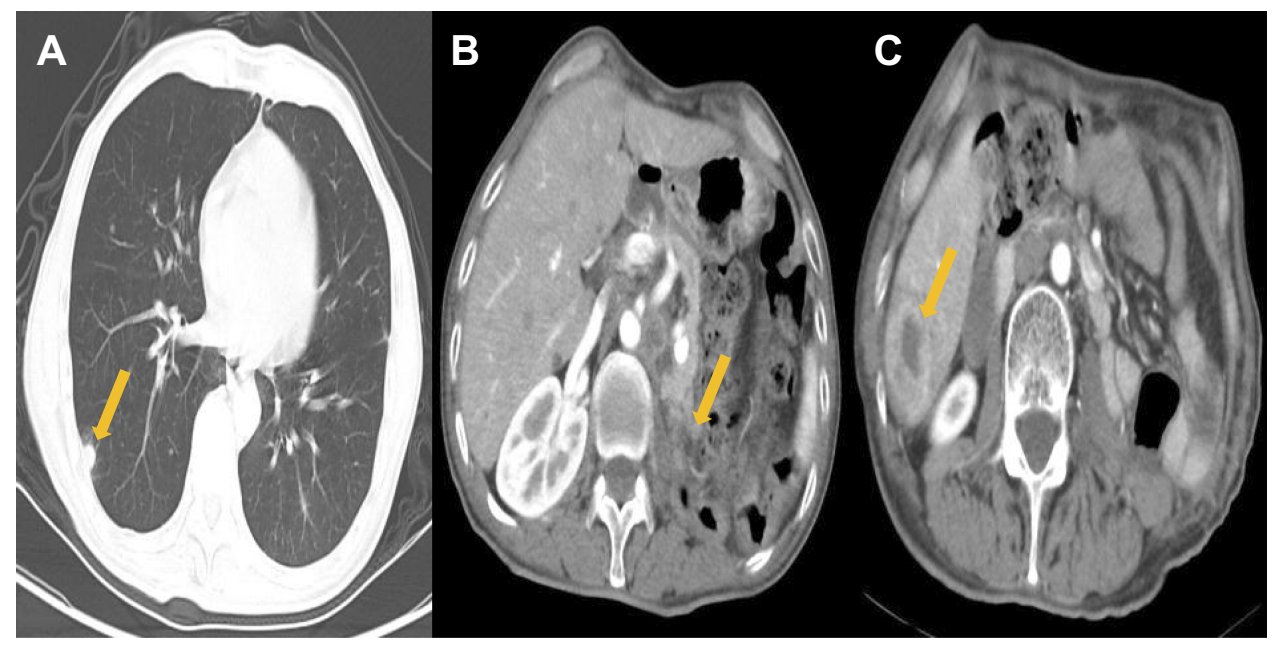

Figure 5 Metastasis revealed in August 2015. Computed tomography of the chest and abdomen in August 2015. (A) Right pulmonary lobes with metastatic nodules, (B) Diffuse enlargement of retroperitoneal lymph nodes and (C) Space-occupying lesion of the liver.

include the prostate (35\%), the lung (18\%), the skin melanoma $(11 \%)$ and the kidney $(9 \%) .{ }^{5}$ Metastatic tumors involving the testis from gastric, colon, pancreatic and thyroid carcinoma have been described in the literature. Luigi Cormio $^{3}$ and Meacham $\mathrm{RB}^{6}$ reported testicular metastasis as the first clinical manifestation of pancreatic adenocarcinoma and colon carcinoma. Appetecchia et $\mathrm{al}^{7}$ described the only case of metastatic medullary thyroid carcinoma involving the testis.
Testicular neoplasms account for only $1 \%$ of all malignancies, and the majority of testicular neoplasms are primary tumors. ${ }^{8}$ There are no specific characteristics of metastatic testicular tumors on imaging data. Metastatic tumors usually present as solitary, unilateral nodules and may mimic primary neoplasms of the testis. ${ }^{9}$

Distinguishing testicular metastasis from primary testicular carcinoma based on ultrasound findings may be very 
difficult. The diagnosis of metastatic lesions of the testis could be defined by spine biopsy of the lesion, but this diagnostic approach might lead to transfixion-mediated tumor metastasis, and the local recurrence rate would increase significantly. Some cases may show histological characteristics indicative of possible metastasis. Medical history is also crucial for differential diagnosis. In conclusion, secondary testicular metastasis should be diagnosed by a comprehensive analysis of the medical history, scrotum ultrasound findings and pathological diagnosis.

Yang et $\mathrm{al}^{10}$ reported that metastasis to the testicle is a rare event because of the strong capsule around the testis termed the "tunica albuginea". In addition, a lower temperature in the scrotum is unfavorable for the growth of metastatic tumor cells, which could explain the relative rarity of testicular metastasis. ${ }^{11}$ Various metastatic routes, including direct invasion, retrograde venous embolism, arterial embolization, retrograde lymphatic extension from para-aortic lymph nodes, transperitoneal seeding and retrograde extension from the vas deferens, have been described as leading to the development of testicular metastasis. ${ }^{8}$ The most common mechanism of tumor spread in urothelial carcinoma (UC) is the lymphovascular system. ${ }^{1}$ However, the molecular mechanisms of the blood-testis and blood-epididymis barriers for preventing metastatic spread to the testis and how metastases overcome these protective mechanisms remain unclear. ${ }^{7}$

A few case reports describing UC metastasis to the testis are available in the literature. All of the cases in those reports of metastatic UC to the testis had tumor origins in the bladder or prostatic urethra. ${ }^{1,4,12}$ Only two case reports are available in the present literature regarding UTUC metastasizing to the testis. ${ }^{1,13}$ The presented case indicates an unusual biological behavior of UTUC for the third time. Although radical nephroureterectomy (with bladder cuff) is the most effective choice for the treatment of renal pelvis carcinoma, there are still few good therapeutic effects for patients with multiple metastases, and the prognosis of these patients is usually poor. Unfortunately, the overall situation of this patient deteriorated gradually, and serial testicular, lung, liver and retroperitoneal lymph node metastasis from urothelial cell carcinoma of the renal pelvis occurred almost simultaneously within one year after the surgery.

Chemotherapy is the main treatment for metastatic testicular cancer for slowing the further development of cancer. According to NCCN (National Comprehensive Cancer Network) guidelines, different chemotherapy schemes are set for the treatment of metastatic testicular cancer. Based on the dose administered, they can be divided into routine and high-dose chemotherapy. The routine path includes the VeIP (vinblastine, ifosfamide, cisplatin) scheme and the TIP (paclitaxel, ifosfamide, cisplatin) scheme. The specific methods of the VeIP scheme are as follows: vinblastine $0.11 \mathrm{mg}$ / $\mathrm{kg}$ intravenous infusion on days $1-2$, ifosfamide $1200 \mathrm{mg} / \mathrm{m}^{2}$ intravenous infusion on days 1-5, and ifosfamide intravenous infusion on days $1-5$. For the first 15 mins, the last 4 hrs and $8 \mathrm{hrs}$, the patient is infused with mesna $240 \mathrm{mg} / \mathrm{m}^{2}$, and on the first to the fifth days with cisplatin $20 \mathrm{mg} / \mathrm{m}^{2}$ intravenously once every 21 days. $^{14}$ The TIP regimen includes intravenous infusion of paclitaxel $250 \mathrm{mg} / \mathrm{m}^{2}$ on the first day, intravenous infusion of ifosfamide $1500 \mathrm{mg} / \mathrm{m}^{2}$ on the second to fifth days, intravenous infusion of mesna $300 \mathrm{mg} / \mathrm{m}^{2}$ before ifosfamide, and then intravenous infusion of ifosfamide for four hours on the second to fifth days. Mesna was administered for $8 \mathrm{hrs}$, and cisplatin $25 \mathrm{mg} / \mathrm{m}^{2}$ intravenous infusion was repeated every 21 days from the 2nd to the 5 th day. ${ }^{15}$ The high-dose chemotherapy regimen consists of an intravenous infusion of carboplatin $700 \mathrm{mg} / \mathrm{m}^{2}$, an intravenous infusion of etoposide $750 \mathrm{mg} / \mathrm{m}^{2}$, and an intravenous infusion of peripheral blood stem cells 5,4 , and 3 days before infusion for two cycles. ${ }^{16}$ In addition, another regimen could be used that includes intravenous infusion of paclitaxel $200 \mathrm{mg} / \mathrm{m}^{2}$ for $24 \mathrm{hrs}$ on the first day, intravenous infusion of ifosfamide $2000 \mathrm{mg} / \mathrm{m}^{2}$ for $24 \mathrm{hrs}$ on the second to fourth day, and protection with methionine sodium at the same time. Sexual therapy was repeated every 14 days for 2 cycles, followed by an intravenous infusion of carboplatin AUC7-8 for 60 mins on days 1-3, an intravenous infusion of etoposide $400 \mathrm{mg} / \mathrm{m}^{2}$ on days $1-3$, and peripheral blood stem cell support during the 14-21 day interval for 3 cycles. ${ }^{17}$ Clinically, different chemotherapy schemes are applied according to different conditions in different patients to achieve the greatest benefit.

The case we present here is just a case report, and the follow-up period was still very short. As with metastatic involvement to other uncommon sites, comprehensive data should be enrolled in a timely manner, especially in patients with invasive primary carcinoma. Simultaneously, timely followup contributed to the diagnosis of these patients who developed the presence of metastatic symptoms. We hope that more cases concerning testis metastasis are reported along with the use of chemotherapy and its clearly revealed benefit.

\section{Acknowledgments}

This work was supported by the National Natural Science Foundation of China (Grant nos. 81372732), the Program for 
Gansu Provincial Health Industry Research (Grant nos. GWGL2013-30) and the Central Universities Natural Science Fundamental Research Project of China (Grant nos. lzujbky-2014-166).

\section{Author Contributions}

All authors made substantial contributions to conception and design, acquisition of data, or analysis and interpretation of data; took part in drafting the article or revising it critically for important intellectual content; gave final approval of the version to be published; and agree to be accountable for all aspects of the work.

\section{Disclosure}

The authors report no conflicts of interest in this work.

\section{References}

1. Manav AN, Kazan E, Ertek MS, Amasyali AS, Culhaci N, Erol H. A rare cause of testicular metastasis: upper tract urothelial carcinoma. Case Rep Urol. 2014;2014:759858.

2. Shinagare AB, Ramaiya NH, Jagannathan JP, Fennessy FM, Taplin ME, Van den Abbeele AD. Metastatic pattern of bladder cancer: correlation with the characteristics of the primary tumor. AJR Am J Roentgenol. 2011;196(1):117-122. doi:10.2214/AJR.10.5036

3. Cormio L, Sanguedolce F, Massenio P, Di Fino G, Bruno M, Carrieri G. Testicular metastasis as the first clinical manifestation of pancreatic adenocarcinoma: a case report. $J$ Med Case Rep. 2015;9:139. doi:10.1186/s13256-015-0626-4

4. Turo R, Smolski M, Hatimy U, et al. A rare case of testicular metastasis of bladder transitional cell carcinoma. Can Urol Assoc J. 2014;8 (3-4):E181-E183. doi:10.5489/cuaj.1690

5. Li B, Cai H, Kang ZC, Wu H, Hou JG, Ma LY. Testicular metastasis from gastric carcinoma: a case report. World J Gastroenterol. 2015;21 (21):6764-6768. doi:10.3748/wjg.v21.i21.6764

6. Meacham RB, Mata JA, Espada R, Wheeler TM, Schum CW, Scardino PT. Testicular metastasis as the first manifestation of colon carcinoma. $J$ Urol. 1988;140(3):621-622. doi:10.1016/S00225347(17)41740-X
7. Appetecchia M, Barnabei A, Pompeo V, et al. Testicular and inguinal lymph node metastases of medullary thyroid cancer: a case report and review of the literature. BMC Endocr Disord. 2014;14:84. doi:10.1186/1472-6823-14-84

8. Buck DA, Byrd RH, Holmes CL, Pollock T. Testicular metastasis in a case of squamous cell carcinoma of the lung. Case Rep Oncol. 2015;8(1):133-137. doi:10.1159/000380814

9. Ulbright TM, Young RH. Metastatic carcinoma to the testis: a clinicopathologic analysis of 26 nonincidental cases with emphasis on deceptive features. Am J Surg Pathol. 2008;32(11):1683-1693. doi:10.1097/PAS.0b013e3181788516

10. Yang KC, Chao Y, Luo JC, et al. The unusual presentation of gastric adenocarcinoma as a testicular mass: a favorable response to docetaxel and cisplatin plus oral tegafur/uracil and leucovorin. J Chin Med Assoc. 2010;73(2):88-92. doi:10.1016/S1726-4901(10) 70007-1

11. Verma N, Babu S, Kushwaha JK, Singhai A. Testicular metastasis of colorectal carcinoma: an unusual presentation. BMJ Case Rep. 2013;2013:bcr2012007849.

12. Kim SO, Choi YD, Jung SI, et al. Prostate cancer with solitary metastases to the bilateral testis. Yonsei Med J. 2011;52(2):362-364. doi:10.3349/ymj.2011.52.2.362

13. Wang $\mathrm{CN}$, Huang $\mathrm{CN}$, Hsiao $\mathrm{PH}$, Chou $\mathrm{YH}$, Huang $\mathrm{CH}$. An unusual testicular metastasis from transitional cell carcinoma of the ureter: a case report. Gaoxiong Yi Xue Ke Xue Za Zhi. 1995;11 (10):578-581.

14. Loehrer PS, Lauer R, Roth BJ, Williams SD, Kalasinski LA, Einhorn LH. Salvage therapy in recurrent germ cell cancer: ifosfamide and cisplatin plus either vinblastine or etoposide. Ann Intern Med. 1988;109(7):540-546. doi:10.7326/0003-4819-1097-540

15. Kondagunta GV, Bacik J, Donadio A, et al. Combination of paclitaxel, ifosfamide, and cisplatin is an effective second-line therapy for patients with relapsed testicular germ cell tumors. $J$ Clin Oncol. 2005;23(27):6549-6555. doi:10.1200/JCO.2005. 19.638

16. Einhorn LH, Williams SD, Chamness A, Brames MJ, Perkins SM, Abonour R. High-dose chemotherapy and stem-cell rescue for metastatic germ-cell tumors. $N$ Engl $J$ Med. 2007;357(4):340-348. doi:10.1056/NEJMoa067749

17. Feldman DR, Sheinfeld J, Bajorin DF, et al. TI-CE high-dose chemotherapy for patients with previously treated germ cell tumors: results and prognostic factor analysis. J Clin Oncol. 2010;28 (10):1706-1713. doi:10.1200/JCO.2009.25.1561
OncoTargets and Therapy

\section{Publish your work in this journal}

OncoTargets and Therapy is an international, peer-reviewed, open access journal focusing on the pathological basis of all cancers, potential targets for therapy and treatment protocols employed to improve the management of cancer patients. The journal also focuses on the impact of management programs and new therapeutic agents and protocols on patient perspectives such as quality of life, adherence and satisfaction. The manuscript management system is completely online and includes a very quick and fair peer-review system, which is all easy to use. Visit http://www.dovepress.com/ testimonials.php to read real quotes from published authors. 